\title{
Permeability of boar and bull spermatozoa to the nucleic acid stains propidium iodide or Hoechst 33258, or to eosin: accuracy in the assessment of cell viability
}

\author{
B. Pintado ${ }^{1}$, J. de la Fuente ${ }^{1}$ and E. R. S. Roldan ${ }^{2 *}$ \\ ${ }^{1}$ Departamento de Reproducción Animal y Conservación de Recursos Zoogenéticos, INIA, 28040-Madrid, Spain; and ${ }^{2}$ Museo Nacional de \\ Ciencias Naturales, CSIC, c/José Gutiérrez Abascal 2, 28006-Madrid, Spain
}

\begin{abstract}
This study was designed to assess whether nucleic acid stains such as propidium iodide and Hoechst 33258 and the cytosolic stain eosin identified equivalent proportions of non-viable cells. Sub-samples of boar spermatozoa stored for up to $72 \mathrm{~h}$, and frozen bull spermatozoa stored in straws and thawed before staining, were exposed to either propidium iodide or Hoechst 33258 alone or in combination. Additional sub-samples were stained with eosin-nigrosin and subsequently with Giemsa. The proportion of non-viable cells identified by propidium iodide alone was equivalent to that observed when it was used in combination with the other fluorescent probe. Similar results were observed for Hoechst 33258. However, direct microscopic examination of sub-samples exposed to both stains revealed that a proportion of spermatozoa stained with propidium iodide did not incorporate Hoechst 33258. This was found consistently in boar and bull spermatozoa under the different experimental conditions used. Quantification showed that the proportion of propidium iodide-positive cells was significantly higher than Hoechst 33258-positive cells. Furthermore, the proportion of propidium iodide-positive cells was higher than cells stained with eosin, but no differences were found between the number of cells stained with Hoechst 33258 or eosin. The proportion of cells stained with propidium iodide was positively correlated with the proportion stained with either Hoechst 33258 or eosin, despite the observation that more cells incorporated propidium iodide. Taken together, these results indicate that there are differences in the ability of fluorescent probes to identify non-viable sperm cells and that this should be considered when staining protocols are used to analyse sperm viability, or when viability is used as a discriminating factor in functional studies, such as those related to acrosomal exocytosis.
\end{abstract}

\section{Introduction}

One of the most important parameters in the evaluation of fertility in a particular male, or in the assessment of methods of semen preservation, is the analysis of sperm viability. In addition, studies of sperm function (for example, acrosomal exocytosis, the so-called 'acrosome reaction') require that quantification of the number of cells responding to physiological stimuli or other reagents is restricted only to viable cells. The inclusion of non-viable cells will bias the results and confound the effect of compounds under investigation.

Several methods have been used to distinguish between viable and non-viable cells, such as the nigrosin-eosin stain (Hancock, 1951), and the fluorescent probes carboxyfluorescein diacetate, propidium iodide (Garner et al., 1986; Harrison and Vickers, 1990) and Hoechst 33258 (De

${ }^{*}$ Correspondence.

Received 18 May 1999
Leeuw et al., 1991). It has been argued that some of these methods are unreliable because, in some species, sperm cells show partial staining (eosin-nigrosin: Hancock, 1957) or because they may overestimate the number of non-viable spermatozoa (fluorescent probes: Tamuli and Watson, 1994).

Methods have also been developed to distinguish, in the same preparation, between spermatozoa that are viable or non-viable, and the integrity of the acrosome (reviewed in Cross and Meizel, 1989). One such method is the triple-stain technique which uses trypan blue, Bismark brown and rose bengal (Talbot and Chacon, 1981), but this method has some disadvantages: it is cumbersome, it overestimates non-viable cells, and it is highly variable between laboratories (Mortimer et al., 1990). Staining with eosin-nigrosin has been combined with Giemsa (Tamuli and Watson, 1994), and this method is reliable and simple enough for routine work (Tamuli and Watson, 1994; Cassinello et al., 1998). In addition, nucleic acid stains such as Hoechst 33258 have been combined with fluoresceinated lectins (Mortimer et al., 1990; Casey et al., 1993; Valcarcel et al., 1997), or with other 
fluorescent probes such as chlortetracycline, to assess capacitation and acrosomal exocytosis (Fraser et al., 1995; Wang et al., 1995). These studies have relied on microscopic examination of spermatozoa using either bright field illumination (that is, triple-stain, eosin-nigrosin and Giemsa) or a combination of phase-contrast optics and epifluorescence. In addition, flow cytometry has been used to characterize populations of spermatozoa rapidly on the basis of at least two attributes. Thus, a combination of fluorescent probes can be used to assess sperm viability (Garner et al., 1986). Alternatively, by combining a probe that distinguishes between viable and non-viable cells (for example propidium iodide) with another that stains the plasma membrane, acrosomal contents or outer acrosomal membrane (for example fluoresceinated lectins), both sperm viability and acrosomal status can be assessed (Tao et al., 1993; Maxwell and Johnson, 1997; Cooper and Yeung, 1998; Harkema et al., 1998).

Typically, flow cytometry studies have included propidium iodide to assess viability, whereas microscopic evaluation has relied on the use of Hoechst 33258. It has been assumed that all nucleic acid stains yield equivalent information, but this assumption has not been examined in detail. In a preliminary series of experiments aimed at studying capacitation and the acrosome reaction in response to a variety of conditions and agents, the ability of propidium iodide, Hoechst 33258 and eosin-nigrosin (together with Giemsa) to detect non-viable cells was compared. The proportion of non-viable cells identified using the three methods was not the same. Therefore, the aim of the present study was to quantify the ability of various staining protocols to identify viable and non-viable sperm cells.

\section{Materials and Methods}

\section{Reagents}

Chemicals were of analytical grade and were purchased from Sigma, BDH or Merck (all of Madrid). Propidium iodide and Hoechst 33258 were from Sigma; nigrosin (CI 50420) was from Merck; eosin yellowish (CI 45380) was from Panreac (Barcelona); and Giemsa was from BDH.

\section{Storage and handling of spermatozoa}

Boar spermatozoa were stored in the Beltsville extender

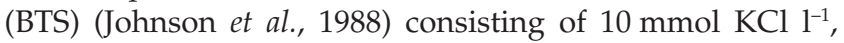
$20.4 \mathrm{mmol}$ trisodium citrate $\left(2 \mathrm{H}_{2} \mathrm{O}\right) \mathrm{l}^{-1}, 15 \mathrm{mmol} \mathrm{NaHCO}_{3} \mathrm{l}^{-1}$, $3.36 \mathrm{mmol}$ EDTA (disodium salt) $1^{-1}, 205 \mathrm{mmol}$ glucose $1^{-1}$ and $50 \mu \mathrm{g}$ kanamycin monosulphate $\mathrm{ml}^{-1}$ and, once semen was added, the $\mathrm{pH}$ was 7.0-7.4.

The standard saline medium used consisted of $142 \mathrm{mmol}$ $\mathrm{NaCl} \mathrm{l}^{-1}, 2.5 \mathrm{mmol} \mathrm{KOH} \mathrm{l}^{-1}, 10 \mathrm{mmol}_{\text {glucose }}{ }^{-1}$ and $20 \mathrm{mmol}$ Hepes $1^{-1}$, adjusted to $\mathrm{pH} 7.55$ at $20^{\circ} \mathrm{C}$ with $\mathrm{NaOH}$. This medium also contained $1 \mathrm{mg}$ poly(vinyl alcohol) $\mathrm{ml}^{-1}$ and $1 \mathrm{mg}$ polyethylene glycol $\mathrm{ml}^{-1}$ and the osmolality was $305 \mathrm{mOsm} \mathrm{kg}{ }^{-1}$. A modified Tyrode's medium was also used, consisting of $120 \mathrm{mmol} \mathrm{NaCl} \mathrm{l}^{-1}, 3.1 \mathrm{mmol} \mathrm{KCl}^{-1}, 2 \mathrm{mmol}$
$\mathrm{CaCl}_{2} \mathrm{l}^{-1}, 0.4 \mathrm{mmol} \mathrm{MgSO}_{4} \mathrm{l}^{-1}, 20 \mathrm{mmol} \mathrm{Hepes} \mathrm{l}^{-1}, 5 \mathrm{mmol}$ glucose $\mathrm{l}^{-1}, 21.7 \mathrm{mmol}$ sodium lactate $\mathrm{l}^{-1}, 1 \mathrm{mmol}$ sodium pyruvate $\mathrm{l}^{-1}, 20 \mathrm{\mu g}$ phenol red $\mathrm{ml}^{-1}$ and $5 \mathrm{mg} \mathrm{BSA} \mathrm{ml}^{-1}$. The medium had a $\mathrm{pH} 7.58$ (adjusted with $\mathrm{NaOH}$ ) and an osmolality of $295 \mathrm{mOsm} \mathrm{kg} \mathrm{m}^{-1}$.

Sperm-rich fractions of semen were collected from Large White boars and were kept at $37^{\circ} \mathrm{C}$ and filtered through gauze. The semen was diluted with BTS (final sperm concentration approximately $1 \times 10^{8}$ cells $\mathrm{ml}^{-1}$ ) at $37^{\circ} \mathrm{C}$, slowly cooled to $17^{\circ} \mathrm{C}$ and maintained at this temperature for up to $72 \mathrm{~h}$.

Bull spermatozoa (Holstein-Friesian) from a commercial company (Aberekin S.A., Derio, Vizcaya), frozen in $0.25 \mathrm{ml}$ straws, were thawed for $30 \mathrm{~s}$ at $37^{\circ} \mathrm{C}$, transferred to an Eppendorf tube and motility was examined. Thawed samples with $\geqslant 40 \%$ motility were used for experiments.

\section{Staining with fluorochromes or eosin-nigrosin and Giemsa}

Aliquots of sperm suspension were stained with propidium iodide, Hoechst 33258, propidium iodide and Hoechst 33258 or with eosin-nigrosin. A stock of Hoechst 33258 (Sigma) was prepared at a concentration of $1 \mathrm{mg} \mathrm{ml}^{-1}$ in distilled water and aliquots were stored frozen at $-20^{\circ} \mathrm{C}$ in the dark. For use in the experiments, the stock solution was diluted 1:10 with modified Tyrode's medium, stored at $5^{\circ} \mathrm{C}$ shielded from light, and used within 1 week. For staining, spermatozoa were diluted in saline medium and the working Hoechst 33258 solution was added, so that the final concentration was $1 \mu \mathrm{g} \mathrm{ml}^{-1}$. The sperm suspension was incubated for $3 \mathrm{~min}$ at room temperature and spermatozoa were immobilized with formaldehyde (final concentration: 0.003\% (w/v); Dott and Foster, 1975; Dott et al., 1975; Harrison and Vickers, 1990). BSA stock solution (5 $\mu \mathrm{l} \mathrm{of} 100 \mathrm{mg} \mathrm{ml}^{-1}$ ) was also added to avoid spermatozoa sticking to the glass and producing staining artefacts. The stained suspension $(10 \mu \mathrm{l})$ was placed between a slide and a coverslip and sealed with nail varnish.

For propidium iodide staining, a procedure modified from Harrison and Vickers (1990) was used. A stock solution of $0.5 \mathrm{mg}$ propidium iodide $\mathrm{ml}^{-1}$ water was prepared, aliquoted and stored frozen at $-20^{\circ} \mathrm{C}$ in the dark. Spermatozoa were diluted in the saline medium and the propidium iodide stock solution was added, so that the final concentration was $5 \mu \mathrm{g} \mathrm{ml}^{-1}$. The suspension was incubated for $5 \mathrm{~min}$ at room temperature and spermatozoa were immobilized with formaldehyde; BSA was added and stained samples were placed between a slide and coverslip as described above.

Spermatozoa were also stained simultaneously with propidium iodide and Hoechst 33258. Spermatozoa suspended in saline medium were exposed to both stains at the concentrations described above, incubated at room temperature for $5 \mathrm{~min}$, and immobilized and mounted as indicated above.

The eosin-nigrosin solution was prepared as described by Tamuli and Watson (1994). Briefly, $10 \mathrm{~g}$ nigrosin was dissolved in distilled water by boiling, and filtered into a cylinder containing $0.7 \mathrm{~g}$ eosin, $7.5 \mathrm{ml}$ of $50 \mathrm{mmol}$ glucose $\mathrm{l}^{-1}$, and $7.5 \mathrm{ml}$ tartrate phosphate buffer (TPB) (50 mmol 
$\mathrm{Na}_{2} \mathrm{HPO}_{4} \mathrm{l}^{-1}, 25 \mathrm{mmol} \mathrm{KH}_{2} \mathrm{PO}_{4} \mathrm{l}^{-1}, \quad 77 \mathrm{mmol}$ potassium sodium tartrate $\mathrm{l}^{-1}$ ), and the volume made up to $100 \mathrm{ml}$. The solution was kept at $5^{\circ} \mathrm{C}$. Staining was carried out by mixing an aliquot of spermatozoa suspended in saline medium with eosin-nigrosin solution (1:3 dilution) for $30 \mathrm{~s}$ before preparing a smear and drying on a warm plate at $37^{\circ} \mathrm{C}$. Smears were fixed in $4 \%(\mathrm{w} / \mathrm{v})$ formaldehyde in TPB, rinsed in slow running water for $10 \mathrm{~min}$, and with distilled water, and stained with Giemsa (Tamuli and Watson, 1994). The Giemsa stain solution consisted of $4.5 \mathrm{ml}$ Giemsa stock solution (Watson, 1975), $3 \mathrm{ml}$ TPB and $32.5 \mathrm{ml}$ distilled water, and was prepared fresh before use. After staining for $90 \mathrm{~min}$, slides were rinsed with distilled water, dried under air and mounted with Permount (Fisher Scientific, Fair Lawn, NJ).

\section{Experimental design}

In Expt 1, boar spermatozoa from 12 different ejaculates, collected and diluted as described, were incubated in BTS for up to $72 \mathrm{~h}$ and assessed for sperm viability. For each ejaculate, two sub-samples were stained with Hoechst 33258, two sub-samples with propidium iodide, and a further two sub-samples with both Hoechst 33258 and propidium iodide. In addition, two sub-samples from each ejaculate were stained with eosin-nigrosin, smeared, dried and stained with Giemsa, as indicated above. When the fluorescent stains were used, spermatozoa were observed within $20 \mathrm{~min}$ of preparation to avoid staining artefacts which could result in an overestimation of non-viable cells. Spermatozoa found to be partially stained with any of the stains were regarded as non-viable cells.

For Expt 2, the number of non-viable cells present in the sample was deliberately increased to mimic potential experimental situations and to test the sensitivity of the staining protocols to detect non-viable cells. Boar spermatozoa from 12 different ejaculates were incubated in BTS for up to $72 \mathrm{~h}$ and, before cell viability was assessed, were mixed 1:1 with a suspension in which the spermatozoa had been treated with three cycles of freezing to $-20^{\circ} \mathrm{C}$ and thawing to room temperature to increase the number of non-viable cells. Samples were stained and analysed as described for Expt 1.

In Expt 3, frozen bull spermatozoa from 12 different ejaculates were thawed as indicated by the supplier and samples with at least $40 \%$ motility were used. Samples were processed as indicated in Expt 1.

\section{Statistical analyses}

For each preparation, 200 spermatozoa were counted, and the percentage of stained cells was transformed $(x=\arcsin$ $\checkmark$ percentage of spermatozoa / 100) to obtain a normal distribution.

In all experiments, statistical differences between the number of stained cells observed after staining with each fluorescent probe alone (that is Hoechst 33258 or propidium iodide) and those obtained when spermatozoa were stained with both probes simultaneously were determined using Student's $t$ test. No differences were found in any of the experiments, and because both estimates corresponded to the same sample, further analysis of the number of Hoechst 33258-stained cells in each sample was calculated as the mean of stained cells in the Hoechst 33258 only sample and the Hoechst 33258-stained cells in the sample in which both probes were used. The same procedure was followed to calculate the number of propidium iodide-positive cells.

Within each experiment, a one-way ANOVA was performed to determine whether there were significant differences among the three staining procedures and, when statistical differences were observed, treatments were compared with the Student-Newman-Keuls method for pairwise multiple comparison. Single linear regression analyses between combinations of the three methods were also carried out. All calculations were performed with the software Sigma Stat v.1.0 (Jaendel Scientific, San Rafael, CA).

\section{Results}

\section{Staining of boar and bull spermatozoa with fluorescent probes or eosin-nigrosin and Giemsa}

When boar or bull spermatozoa were incubated with either Hoechst 33258 or propidium iodide, a proportion of cells incorporated the stain and were readily identified by fluorescent microscopy. When spermatozoa were simultaneously incubated with the two fluorescent probes and examined with their respective microscope filters, it was apparent that some cells stained with propidium iodide did not stain with Hoechst 33258 (Fig. 1). This was observed in spermatozoa from both species and in all semen samples or frozen and stored samples. The number of propidium iodide-stained cells was consistently greater than the number of Hoechst 33258-stained cells (see below).

When boar or bull spermatozoa were incubated with eosinnigrosin, smeared and stained with Giemsa, spermatozoa could be distinguished easily on the basis of the absence or presence of a stained post-acrosomal region (Fig. 2). Preliminary experiments indicated that the number of stained cells did not differ when they were stained solely with eosin-nigrosin or when they were stained first with eosin-nigrosin and then with Giemsa. Furthermore, washing of smears before staining with Giemsa did not remove the eosin stain, which is in agreement with the findings of Tamuli and Watson (1994).

\section{Experiment 1}

In this experiment, 12 boar semen samples were analysed after storage in BTS. Mean values of stained cells obtained with each staining protocol are presented (Fig. 3). Although the number of propidium iodide-stained cells was higher than the number of Hoechst 33258-positive cells, statistical analyses of these data showed no significant differences among the three vital stains examined. This was probably due to the low proportion of stained cells observed with the three stains. When percentages of stained cells were compared by single linear regression (Table 1) a significant 

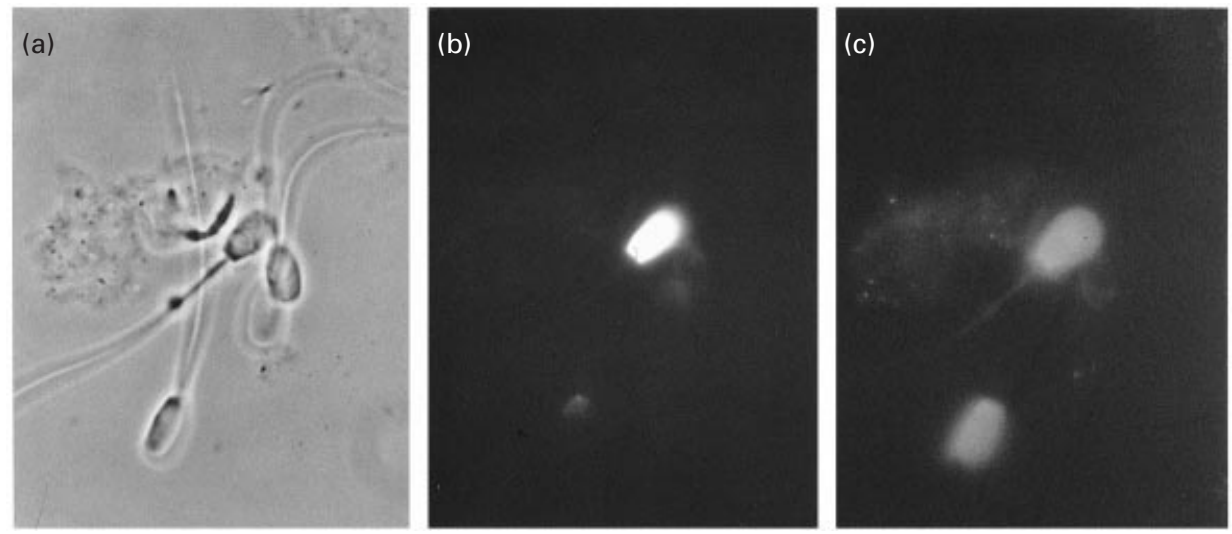

Fig. 1. Boar spermatozoa after staining with Hoechst 33258 and propidium iodide. Spermatozoa were incubated with both Hoechst 33258 (final concentration $1 \mu \mathrm{g} \mathrm{m}^{-1}$ ) and propidium iodide (final concentration $5 \mu \mathrm{g} \mathrm{ml}^{-1}$ ) for $5 \mathrm{~min}$ and examined (a) under phase-contrast optics, (b) using the filter for Hoechst 33258, and (c) using the filter for propidium iodide. Note that with propidium iodide, two spermatozoa are stained, whereas with Hoechst 33258 only one spermatozoon has incorporated the stain.

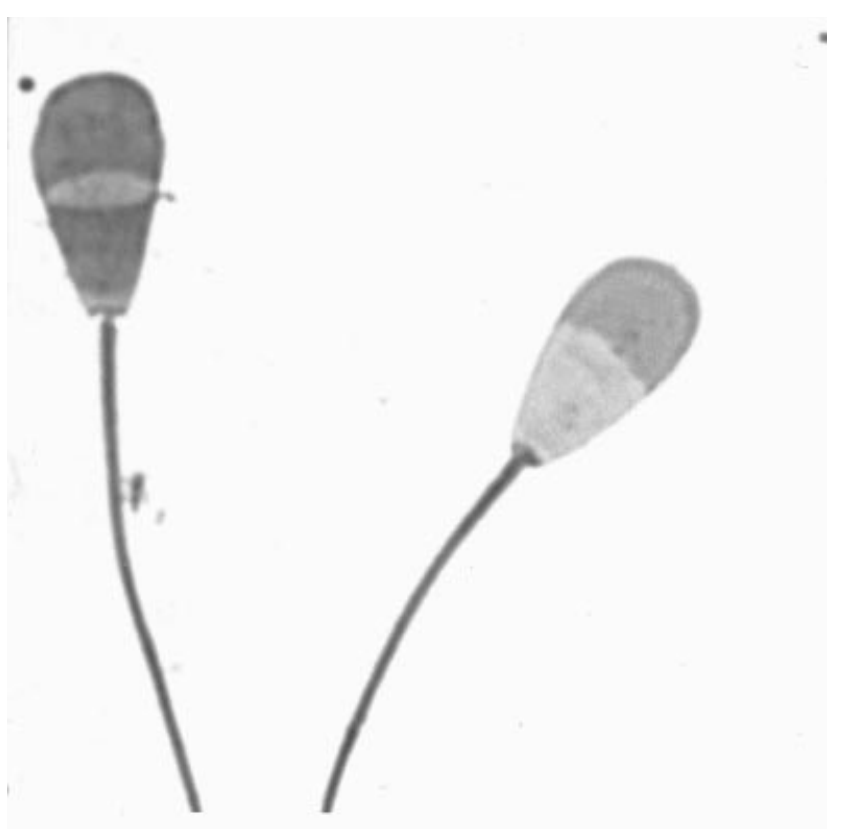

Fig. 2. Bull spermatozoa after staining with eosin-nigrosin and Giemsa. The sperm cell on the left has incorporated eosin, as observed in the post-acrosomal region, whereas the cell on the right has not incorporated the stain. The acrosomal regions are stained with Giemsa.

$(P<0.01)$ correlation was found in all cases; the highest $R$ values corresponded to the comparison between propidium iodide and Hoechst stain $(R=0.964)$.

\section{Experiment 2}

The aim of this experiment was to assess whether the correlation between the three vital stains found with fresh semen was also observed when spermatozoa were subjected

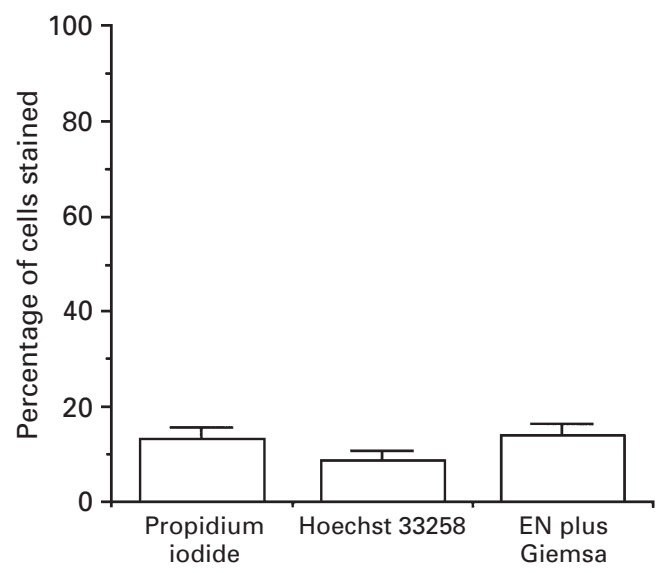

Fig. 3. Staining of stored boar spermatozoa with propidium iodide, Hoechst 33258 or eosin-nigrosin plus Giemsa (EN plus Giemsa). Values are means \pm SEM of 12 different semen samples. Correlation $(R)$ and probability $(P)$ values for comparisons among groups are given in Table 1.

to damage and, thus, whether the number of non-viable cells increased consistently with the three staining protocols. Diluted boar semen was frozen to $-20^{\circ} \mathrm{C}$ and thawed at room temperature three times, and this suspension of non-viable spermatozoa was mixed with an equal volume of spermatozoa from 12 different semen samples that had been stored in BTS for 48-72 h. Under these conditions, staining with propidium iodide resulted in a higher proportion of stained cells $(66.4 \pm 2.4 \%)$ than that observed with Hoechst $33258(60.1 \pm 1.66 \%)$ or eosin-nigrosin and Giemsa $(56.9 \pm 1.84 \%)$. No differences were found between the Hoeschst 33258 and eosin-nigrosin and Giemsa (Fig. 4). Within this experiment, a significant correlation $(R=0.727$, $P<0.01)$ was found between the number of cells stained with propidium iodide and those stained with Hoechst 33528. All other comparisons failed to show any correlation (Table 1). 
Table 1. Correlation $(R)$ and probability $(P)$ values obtained by single linear regression within each experiment comparing three staining procedures for boar and bull spermatozoa

\begin{tabular}{lccc}
\hline & PI $\times$ Hoechst & PI $\times$ EN plus Giemsa & Hoechst $\times$ EN plus Giemsa \\
\hline Expt 1 & & & \\
$R$ & 0.964 & 0.709 & 0.836 \\
$P$ & $<0.0001$ & 0.0099 & 0.0007 \\
Expt 2 & & & \\
$R$ & 0.727 & 0.535 & 0.383 \\
$P$ & 0.0074 & 0.0733 & 0.219 \\
Expt 3 & 0.946 & 0.829 & 0.803 \\
$R$ & $<0.0001$ & 0.0009 & 0.0017 \\
$P$ &
\end{tabular}

For each experiment, 12 semen samples from different boars (Expts 1 and 2) or bulls (Expt 3) were analysed. EN plus Giemsa: eosin-nigrosin plus Giemsa; Hoechst: Hoechst 33258; PI: propidium iodide.

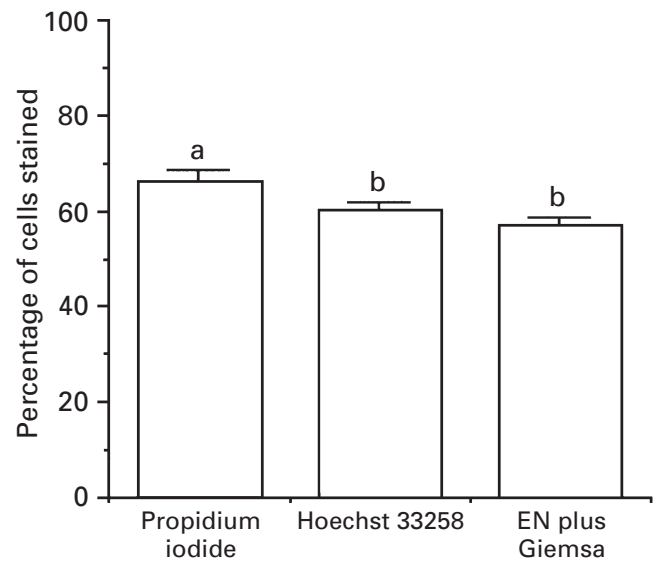

Fig. 4. Staining with propidium iodide, Hoechst 33258 or eosin-nigrosin plus Giemsa (EN plus Giemsa) of a mixture of stored boar spermatozoa and boar spermatozoa treated to three cycles of freezing and thawing to increase the number of non-viable cells. Values are means \pm SEM of 12 different semen samples. Bars with different letters are significantly different $(P<0.05$; ANOVA and Student-Newman-Keuls). Correlation $(R)$ and probability $(P)$ values for comparisons among groups are given in Table 1.

\section{Experiment 3}

Frozen samples from 12 different semen samples were studied to determine whether bull spermatozoa showed significant differences in the number of non-viable cells detected by the three vital stains. In this experiment, a pattern identical to that observed in Expt 2 was found (Fig. $5)$. Propidium iodide gave a significantly higher $(P<0.05)$ proportion of stained cells $(58.0 \pm 4.38 \%)$ than either Hoechst $33258(44.3 \pm 4.57 \%)$ or eosin-nigrosin and Giemsa $(42.8 \pm 3.98 \%)$ and no differences were found between Hoechst 33258 and eosin-nigrosin and Giemsa. The proportion of cells stained with propidium iodide and Hoechst $33258(R=0.946, P=0.0001)$ and those stained with propidium iodide and eosin $(R=0.829, P=0.0009)$ were strongly correlated, but in contrast to Expt 2, Hoechst 33258and eosin-stained cells were also correlated (Table 1, Fig. 6).

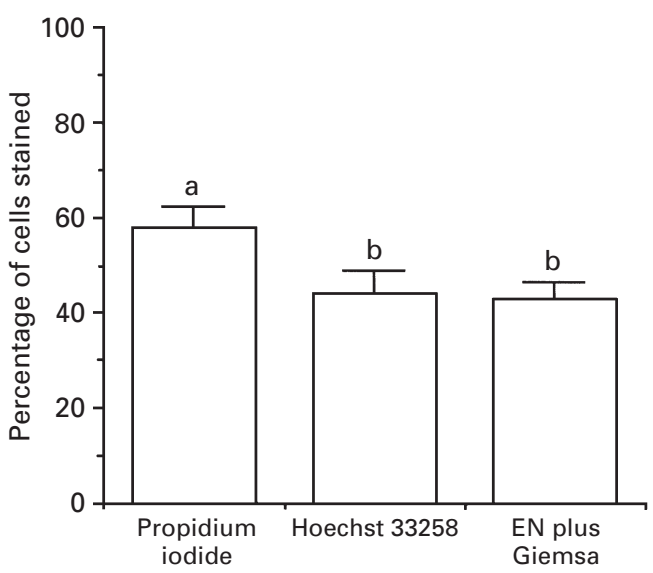

Fig. 5. Staining of frozen and stored bull spermatozoa with propidium iodide, Hoechst 33258 or eosin-nigrosin plus Giemsa (EN plus Giemsa). Values are means \pm SEM of 12 different semen samples. Bars with different letters are significantly different $(P<0.05 ;$ ANOVA and Student-Newman-Keuls). Correlation $(R)$ and probability $(P)$ values for comparisons among groups are given in Table 1.

This may reflect the accuracy of the eosin-nigrosin and Giemsa procedure in bull spermatozoa, leading to more consistent staining patterns compared with boar spermatozoa.

Since bull samples covered a wide range of non-viable cells, data were plotted to determine the differences obtained with the different staining methods. In theory, regression coefficients should be equal to 1 when estimates of nonviable cells agree perfectly. The regression lines obtained from plotting values obtained with propidium iodide and either Hoechst 33258 or eosin were not equal to 1 and, in both cases, the regression lines obtained deviated towards propidium iodide (that is, propidium iodide values were higher than those corresponding to the other two staining methods) (Fig. 6a,b). In the comparison between propidium iodide and Hoechst 33258, the line obtained had a slope similar to the line corresponding to a theoretical regression 

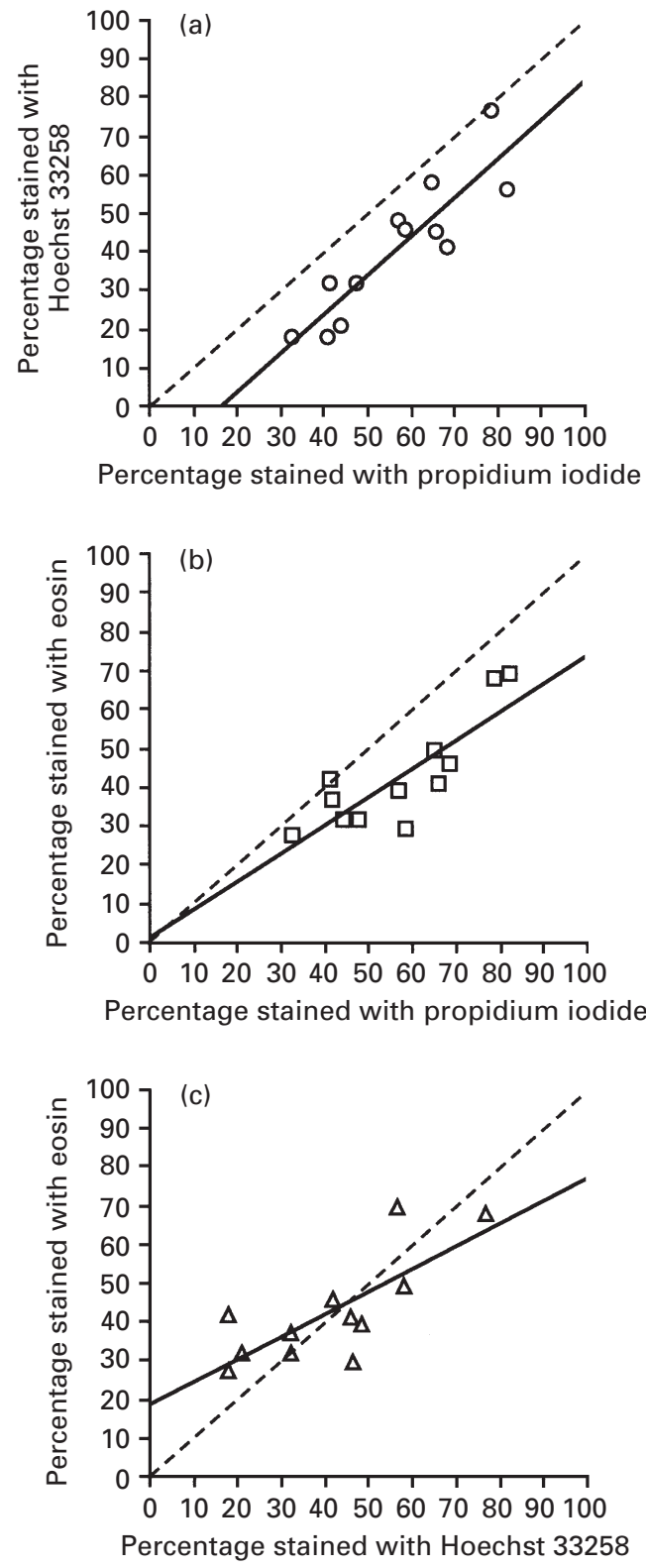

Fig. 6. Correlations between the proportions of frozen and stored bull spermatozoa stained with propidium iodide, Hoechst 33258 or eosin-nigrosin plus Giemsa (EN plus Giemsa). Values correspond to 12 different semen samples. (a) Propidium iodide versus Hoechst $33258\left(y=1.013 x-16.481, r^{2}=0.797, P=0.0001\right)$; (b) propidium iodide versus EN plus Giemsa $\left(y=0.729 x+1.368, r^{2}=0.69\right.$, $P=0.0008)$; and (c) Hoechst versus EN plus Giemsa $(y=0.588 x$ $\left.+18.615, r^{2}=0.579, P=0.004\right)$.

line of 1 , whereas when the comparison was made with eosin the slope was different, and higher differences between the two staining methods were found as the percentage of nonviable cells increased. The comparison between Hoechst 33258 and eosin revealed a different pattern; the regression line showed higher values for eosin when there was a small proportion of non-viable cells and the opposite for a large proportion of non-viable cells.

\section{Discussion}

The results of this study indicate that the proportion of nonviable cells identified by staining with the fluorescent probes propidium iodide or Hoechst 33258, or with the cytosolic stain eosin, may differ depending on which vital stain is used. These differences emphasise the need for careful interpretation of sperm functional studies when these stains are used to distinguish between viable and non-viable cells before quantification of cellular responses, such as the occurrence of the acrosome reaction.

Spermatozoa from both bulls and boars showed consistently that propidium iodide stained more cells than Hoechst 33258 . Hoechst 33258 stained a similar proportion of cells to that observed after staining with the cytosolic stain eosin. Although differences were not statistically significant when boar spermatozoa were stored for 2-3 days in BTS, possibly due to the small proportion of non-viable cells, significant differences were seen when the number of nonviable cells was increased by mixing equal volumes of stored spermatozoa and spermatozoa that had been treated to render them non-viable. Spermatozoa from another species (bulls) in which the proportion of non-viable sperm cells was expected to be high, such as in frozen-thawed samples, were used to confirm this result. In these samples, the proportion of spermatozoa stained with propidium iodide was also higher than after staining with either Hoechst 33258 or eosin.

Spermatozoa were stained with the two fluorescent stains under the same conditions, using each fluorochrome separately or in combination. There was no difference in the proportion of cells stained with propidium iodide when the stain was used alone or in combination with the other fluorochrome. A similar result was found for Hoechst 33258. Thus, inclusion of both probes did not interfere with their staining abilities. However, exposure of spermatozoa to both fluorochromes simultaneously showed, by direct microscopic examination, that some spermatozoa stained with both propidium iodide and Hoechst 33258, whereas other spermatozoa stained only with propidium iodide. The opposite situation, that is spermatozoa stained with Hoechst 33258 and not with propidium iodide, was not observed. In a study on equine spermatozoa, propidium iodide was found to be equivalent to Hoechst 33258 when viability was estimated either by flow cytometry or fluorescent microscopy (Papaioannou et al., 1997). The discrepancy with the results of the present study, in which viability assessments with these two fluorochromes were not equivalent, could be due to differences in methodology or to species differences.

It is possible that one of the fluorochromes stained a smaller number of cells because the conditions were suboptimal. This is unlikely because the conditions used in the present study were validated in other studies on pig and bull spermatozoa (for example Melendrez et al., 1994; Fraser et al., 1995; Wang et al., 1995). In addition, in the present study staining was carried out at room temperature for at least 3-5 min, which is the minimum time required for the cells to take up the maximum amount of stain. Incubation for periods of up to $30 \mathrm{~min}$ with Hoechst 33258 (Cross et al., 1986; de Leeuw et al., 1991) or $130 \mathrm{~min}$ with propidium 
iodide (Harrison and Vickers, 1990) resulted in no further increase in the proportion of stained cells.

Staining with eosin-nigrosin followed a different technical protocol; spermatozoa were exposed to the stain for a few seconds before they were smeared onto slides. However, the proportion of stained cells detected with this protocol was not different from that observed when Hoechst 33258 was used, as also found using human spermatozoa (Mortimer et al., 1990), indicating that both Hoechst 33258 and eosin may estimate the proportion of non-viable cells in sperm populations more accurately than propidium iodide. The proportion of non-viable cells observed after staining with eosin was significantly lower than that observed after propidium iodide, which is in agreement with a study using ram and boar spermatozoa (Tamuli and Watson, 1994).

It is unclear why there are differences in the ability of these stains to stain sperm cells, with propidium iodide consistently staining more spermatozoa than the other two stains. It has been recognized that long incubation periods leading to senescence of cells can create artefacts and increase the number of cells that incorporate the fluorescent probes (Harrison and Vickers, 1990). In the present study, this problem was avoided by incubating spermatozoa with the fluorochromes for a short period of time, but differences among the methods were found. Significant differences were observed in previous studies in which eosin-nigrosin and some fluorescent stains were compared; percentages of viable spermatozoa were higher with the fluorescent stains (Centola et al., 1990; Tamuli and Watson, 1994; Cassinello et al., 1998). One possibility is that some fluorescent probes are slightly toxic to the sperm cell (Woelders, 1991).

The number of cells stained with propidium iodide and the number of spermatozoa stained with Hoechst 33528 was correlated in the three experimental series, despite the fact that significantly more spermatozoa were stained with propidium iodide. Furthermore, propidium iodide also showed a positive correlation with eosin-nigrosin and Giemsa (statistically significant in Expts 1 and 3 and tending to significance in Expt 2), whereas the average proportion of propidium iodide-positive cells was significantly greater. The proportion of cells that stained with eosin (when using the eosin-nigrosin and Giemsa protocol) was significantly correlated with the proportion of cells staining with Hoechst 33258 in bull spermatozoa, but the results were not clear-cut for boar spermatozoa. In some studies, correlations have been used as a basis for determining the superiority of one method over another (for example, see Perez et al., 1997). However, the results of the present study highlight the fact that a positive significant correlation between staining protocols does not necessarily mean that they yield similar results when assessing sperm viability. At this stage it is not posssible to determine which is the most accurate method, that is, the method that estimates most accurately the real proportion of non-viable cells in a sperm sample, due to the lack of a method that gives totally reliable and indisputable results.

Staining with fluorescent probes can be combined with observations by phase-contrast optics for determination of the number of viable spermatozoa along with assessment of acrosomal status. An alternative to this approach is the use of the eosin-nigrosin and Giemsa staining protocol. This staining method could be very useful since it is simpler than that described by Talbot and Chacon (1981) and because it has the advantage that a fluorescent microscope is not required to examine stained spermatozoa. Furthermore, this protocol results in permanent preparations that could be convenient for quality control. One disadvantage is that it requires staining spermatozoa with Giemsa and this could extend the staining protocol by $1-2 \mathrm{~h}$. Whatever the method used, it is important to consider that the choice of vital stain can influence the results of assessment of viability and that this would influence the outcome of quantitative studies of cellular responses such as capacitation or the acrosome reaction.

In conclusion, differences were observed between the ability of different stains to identify non-viable spermatozoa; propidium iodide consistently showed greater numbers of stained cells compared with the other stains. This indicates that in studies focusing, for example, on the viability of spermatozoa after storage, or on functional responses of cells (in which viability is used as a discriminating factor), attention should be given to the possible biases introduced by the vital stains.

This work was supported by grant 07B/0006/1997 from the Comunidad Autónoma de Madrid. The authors thank Montse Gomendio for statistical advice.

\section{References}

Casey PJ, Hillman RB, Robertsonb KR, Yudin AI, Liu IKM and Drobnis EZ (1993) Validation of an acrosomal stain for equine sperm that differentiates between living and dead sperm Journal of Andrology 14 289-297

Cassinello J, Abaigar T, Gomendio M and Roldan ERS (1998) Characteristics of the semen of three endangered species of gazelles (Gazella dama mohrr, G. dorcas neglecta and G. cuvieri) Journal of Reproduction and Fertility 113 35-45

Centola GM, Mattox JH, Burde S and Leary JF (1990) Assessment of viability and acrosome status of fresh and frozen-thawed human spermatozoa using single wave-fluorescence microscopy Molecular Reproduction and Development 27 130-135

Cooper TG and Yeung CH (1998) A flow cytometric technique using peanut agglutinin for evaluating acrosomal loss from human spermatozoa Journal of Andrology 19 542-550

Cross NL and Meizel S (1989) Methods for evaluating the acrosomal status of mammalian sperm Biology of Reproduction 41 635-641

Cross NL, Morales P, Overstreet JW and Hanson FW (1986) Two simple methods for detecting acrosome-reacted human sperm Gamete Research 15 213-226

De Leeuw AM, Den Daas JHG and Woelders H (1991) The fix vital stain method: simultaneous determination of viability and acrosomal status of bovine spermatozoa Journal of Andrology 12 112-118

Dott HM and Foster GC (1975) Preservation of differential staining of spermatozoa by formol citrate Journal of Reproduction and Fertility 45 57-60

Dott HM, Moor RM and Polge C (1975) Artificial insemination with spermatozoa in formaldehyde Journal of Reproduction and Fertility 46277

Fraser LR, Abeydeera LR and Niwa K (1995) $\mathrm{Ca}^{2+}$-regulating mechanisms that modulate bull sperm capacitation and acrosomal exocytosis as determined by chlortetracycline analysis Molecular Reproduction and Development 40 233-241

Garner DL, Pinkel D, Johnson LA and Pace MM (1986) Assessment of spermatozoal function using dual fluorescent staining and flow cytometric analyses Biology of Reproduction 34 127-138

Hancock JL (1951) A staining technique for the study of temperature shock in semen Nature 167323

Hancock JL (1957) The morphology of boar spermatozoa Journal of the Royal Microscopical Society 76 84-96 
Harkema W, Harrison RAP, Miller NGA, Topper EK and Woelders H (1998) Enhanced binding of zona pellucida proteins to the acrosomal region of intact boar spermatozoa in response to fertilizing conditions: a flow cytometric study Biology of Reproduction 58 421-430

Harrison RAP and Vickers SE (1990) Use of fluorescent probes to assess membrane integrity in mammalian spermatozoa Journal of Reproduction and Fertility 88 343-352

Johnson LA, Aalbers JG and Grooten HJG (1988) Artificial insemination of swine: fecundity of boar semen stored in Beltsville TS (BTS), modified Modena (MM) or MR-A and inseminated in one, three and four days after collection Zuchthygiene 23 49-55

Maxwell WMC and Johnson LA (1997) Membrane status of boar spermatozoa after cooling or cryopreservation Theriogenology 48 209-219

Melendrez CS, Meizel S and Berger T (1994) Comparison of the ability of progesterone and heat solubilized porcine zona pellucida to initiate the porcine sperm acrosome reaction in vitro. Molecular Reproduction and Development $39433-438$

Mortimer D, Curtis EF and Camezind AR (1990) Combined use of fluorescent peanut agglutinin lectin and Hoechst 33258 to monitor the acrosomal status and vitality of human spermatozoa Human Reproduction 599-103

Papaioannou KZ, Murphy RP, Monks RS, Hynes N, Ryan MP, Boland MP and Roche JF (1997) Assessment of viability and mitochondrial function of equine spermatozoa using double staining and flow cytometry Theriogenology 48 299-312

Perez LJ, Valcarcel A, de las Heras MA and Baldassarre H (1997)
Comparative study of four techniques for evaluation of sperm quality in ovine and bovine frozen-thawed samples Reproduction in Domestic Animals 32 157-160

Talbot P and Chacon R (1981) A triple stain technique for evaluating normal acrosome reactions of human sperm Journal of Experimental Zoology 215 201-208

Tamuli MK and Watson PF (1994) Use of a simple staining technique to distinguish acrosomal changes in the live sperm sub-population Animal Reproduction Science 35 247-254

Tao J, Critser ES and Critser JK (1993) Evaluation of mouse sperm acrosomal status and viability by flow-cytometry Molecular Reproduction and Development 36 183-194

Valcarcel A, de las Heras MA, Perez L, Moses DF and Baldassarre H (1997) Assessment of the acrosomal status of membrane-intact ram spermatozoa after freezing and thawing, by simultaneous lectin/Hoechst 33258 staining Animal Reproduction Science 45 299-309

Wang WH, Abeydeera LR, Fraser LR and Niwa K (1995) Functional analysis using chlortetracycline fluorescence and in vitro fertilization of frozenthawed ejaculated boar spermatozoa incubated in a protein-free chemically defined medium Journal of Reproduction and Fertility 104 305-313

Watson PF (1975) Use of a Giemsa stain to detect changes in acrosomes of frozen ram spermatozoa Veterinary Record 97 12-15

Woelders H (1991) Overview of in vitro methods for evaluation of semen quality. In Boar Semen Preservation II (Reproduction of Domestic Animals, Supplement 1) pp 145-164 Eds LA Johnson and D Rath. Paul Parey, Berlin 